\title{
ON THE USE OF POSITRONS TO PROBE MAGNETIC VERSUS ELECTROSTATIC TURBULENCE
}

\author{
by \\ R.D. STAMBAUGH
}

This is a preprint of a paper to be presented at the 8th Topical Conference on High Temperature Plasma Diagnostics, May 7-10, 1990, Hyannis, Massachusetts, and to be printed in the Proceedings.

\author{
Work supported by \\ Department of Energy \\ Contract DE-AC03-89ER51114
}

GENERAL ATOMICS PROJECT 3466
OCTOBER 1990

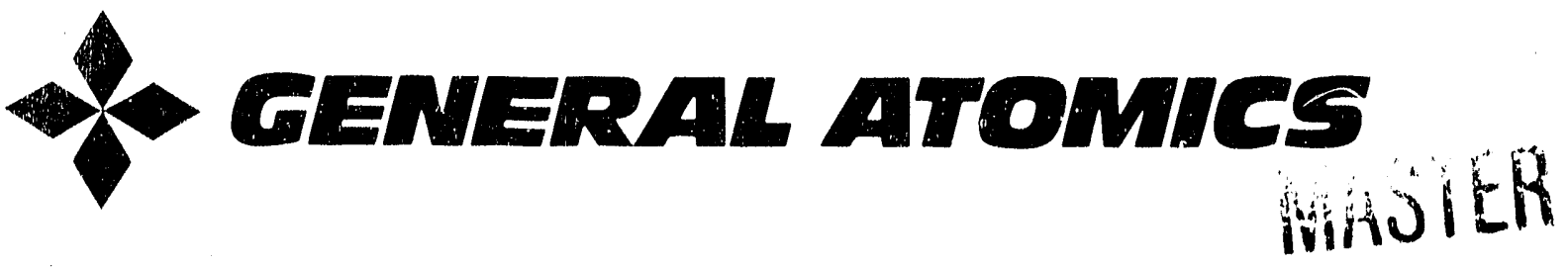




\title{
On the use of positrons to probe magnetic versus electrostatic turbulence
}

\author{
R.D. Stambaugh \\ General Atomics, San Diego, California 92186-9784
}

Kwon, et al. have shown that runaway electron (positron) diffusion is produced by magnetic turbulence and unaffected by electrostatic turbulence. By measuring the diffusion coefficient of positrons at runaway energies $(0.1-2 \mathrm{MeV})$ as a function of radius for two discrete positron energies, the radial correlation length $W$ of the turbulence can be extracted. Then if the thermal electrons are in the weak turbulence regime, the thermal electron diffusion coefficient from magnetic fluctuations alone can be calculated and compared to values from other techniques. We propose to inject charged energetic positrons (100-2000 keV) in few msec bursts from radioactive sources by means of their curvature drift when trapped in toroidal field ripples. The energetic positrons will diffuse over $60-600 \mathrm{msec}$ time scales. At any time the radial profile of the positrons can be sampled by injecting a small solid pellet. A fraction of all the positrons on a flux surface will annihilate in the pellet as it passes that flux surface. The time dependent $0.511 \mathrm{MeV} \gamma$-ray signal then can be unfolded into the positron radial profile and the positron diffusion coefficient determined from the time evolution of those profiles. 


\section{INTRODUCTION}

Recently new vigor has been put into the search for the cause of anomalous cross-field transport in magnetically confined plasmas. A very basic question that remains is whether the transport arises from primarily magnetic or electrostatic fluctuations. What might be called circumstantial evidence ${ }^{1}$ at present favors the electrostatic turbulence hypothesis. The direct resolution of this issue is significantly hampered by the difficulty of making fluctuation measurements in the plasma interior. Nevertheless a number of techniques, e.g., FIR scattering and beam emission spectroscopy for $\tilde{n}$ and the heavy ion beam probe for $\tilde{\phi}$, have been developed to pursue the electrostatic turbulence hypothesis. No corresponding approaches to detecting interior magnetic fluctuations at the required level of $\tilde{B} / \mathrm{B} \sim 10^{-4}$ in large hot plasmas are now available. Hence the pursuit of the magnetic turbulence hypothesis suffers from a lack of direct evidence and must rely on secondary methods.

Among these secondary methods, the radial transport of runaway electrons is a sensitive probe of magnetic turbulence. If we assume that the same turbulence (either magnetic or electrostatic) produces both the transport of thermal and runaway electrons $\left(E_{\mathrm{R}} \sim 100 E_{\mathrm{TH}}\right)$, then the diffusion coefficient for runaway $\chi_{\mathrm{R}}$ will be 1-10 times the bulk plasma $\chi_{\mathrm{TH}}$ for magnetic turbulence but $1 / 100$ to $1 / 10 \chi_{\mathrm{TH}}$ for electrostatic turbulence. Hence the diffusion of runaways can be used to magnify the difference between electrostatic and magnetic turbulence. Although we can be sure that the measured runaway diffusion, however large it is, is caused by magnetic turbulence, we must also show those levels of fluctuations are sufficient to account for the thermal transport. Kwon, Diamond et al. ${ }^{2}$ have developed the theoretical 
formalism necessary to relate runaway transport to thermal transport in their study of runaway transport in ASDEX.

Herein, we propose to improve on that study by using runaway positrons instead of electrons. Since the positrons come from a radioactive source, their energy spectrum and space and time of origin in the plasma can be known much better than for runaway electrons. We also propose to find out at any later time the radial distribution of the positrons by means of their characteristic $2 \gamma(0.511 \mathrm{MeV}$ each $)$ annihilation in an injected solid pellet. If we are allowed a bit of analogy, studies of fluid turbulence have profited immensely from the use of different colors of fluids which, when mixed, react chemically to form a third color. Positrons represent "colored electrons" and they can be made to "light up" with annihilation radiation in a pellet. Runaway electrons, in contrast are hard to pick out from the larger population of thermal electrons. The idea of using positrons as test particles for studying transport is not new. ${ }^{3,4}$ The use of runaway positrons is a new suggestion.

\section{THEORY}

We follow the development of Kwon and Diamond. ${ }^{2}$ The diffusion of thermal electrons in magnetic turbulence is given by

$$
\begin{array}{ll}
\chi_{\mathrm{eTH}}=\left|v_{\|}\right|_{\mathrm{TH}} b_{0} W & \text { strong turbulence } \\
\chi_{\mathrm{eTH}}=\frac{\pi\left|v_{\|}\right|_{\mathrm{TH}} L_{8} b_{0}^{2}}{\bar{k}_{\theta} \bar{W}_{m \mathrm{I}}} \quad \text { weak turbulence }
\end{array}
$$


where $b_{0}$ is the RMS fluctuation amplitude, $L_{s}=R q / \tilde{s}$ is the shear length $\left(\tilde{s}=r q^{\prime} / q\right)$, $\bar{k}_{\theta}$ is the average poloidal wavenumber of the turbulence, and $W \sim \bar{W}_{m \mathrm{I}}$ is the radial correlation length of the turbulence. The diffusion of runaways is similar to the weak turbulence case except for an additional radial structure function $S$ which reduces the $\chi$ owing to the increasing decorrelation of the turbulence and the electron's motion as the excursion of the drift orbit from flux surfaces increases.

For example $S$ might be Gaussian.

$$
\begin{gathered}
\chi_{\mathrm{eR}}=\frac{\pi\left(v_{\|}\right)_{\mathrm{R}} L_{\Delta} b_{0}^{2}}{\bar{k}_{\theta} \bar{W}_{m \mathrm{I}}} e^{-\left[L, v_{\mathrm{DR}} /\left(W v_{\| \mathrm{R}}\right)\right]^{2}} \\
v_{\mathrm{DR}}=\frac{1}{\Omega_{\mathrm{eR}}}\left(v_{\|}^{2}+\frac{1}{2} v_{\perp}^{2}\right)
\end{gathered}
$$

Comparing Eq. (3) to (3), we see that $\chi_{e \mathrm{R}} \simeq(1-10) \chi_{e \mathrm{TH}}$, depending on $S$. On the other hand, for electrostatic turbulence, $\chi_{e \mathrm{R}} / \chi_{\mathrm{eTH}} \simeq\left(v_{\| \mathrm{TH}} / v_{\| \mathrm{R}}\right) S \simeq 1 / 10$ to $1 / 100$. If we can measure $\chi_{e} \mathrm{R}$ for different known runaway energies, we can calculate $W$ from

$$
\left.\frac{\chi_{e R}\left(E_{1}\right)}{\chi_{e R}\left(E_{2}\right)}=\frac{v_{\| R}(1)}{v_{\| R}(2)} e^{-\frac{L_{g}^{2}}{W^{2}}\left\{\left[\frac{v_{D R}}{v_{\| R}(1)}\right]^{2}-\left[\frac{v_{D R}(2)}{v_{\| R}}\right]^{2}\right.}\right\}
$$


Knowing $W$, if we can assume the thermal electrons are in the weak turbulence regime, we can calculate

$$
\chi_{e, \mathrm{TH}}=\frac{v_{\| \mathrm{TH}}}{v_{\| \mathrm{R}}} \chi_{e \mathrm{R}} e^{\left(\frac{L_{g}}{W} \frac{v_{\mathrm{DR}}}{v_{\| \mathrm{R}}}\right)^{2}}
$$

If $\chi_{e}, \mathrm{TH}$ so calculated agrees with power balance results, we will have shown that the thermal transport is accounted for by magnetic turbulence. If the thermal electrons are in the strong turbulence regime then

$$
\chi_{e, \mathrm{TH}}=\frac{\chi_{\mathrm{eR}} v_{\| \mathrm{TH}} \bar{k}_{\theta} W W_{m \mathrm{I}}}{v_{\| \mathrm{R}} \pi L_{a} b_{0}} e^{\left(\frac{L_{e}}{W} \frac{v_{\mathrm{DR}}}{v_{\| \mathrm{R}}}\right)^{2}}
$$

In this case, information on $\bar{k}_{\theta}$ and $b_{0}$ from other diagnostics and/or parameter scans for $\chi_{e \mathrm{R}}(\mathrm{B}, \mathrm{I}, n$, etc.) would be needed to close the problem. 


\section{EXPERIMENTAL CONSIDERATIONS}

\section{A. Introduction of the positrons}

Neutral positronium beams for getting positrons into plasma are being developed. ${ }^{3}$ However, these beams will be of low energy. We have examined ways to get energetic charged positrons into the plasma directly.

Positron sources are available commercially. The sources below have a typical $\beta$ decay spectrum, a roughly symmetric peak about an average energy and with an upper endpoint energy.

$\begin{array}{lccc}\text { Nuclide } & \text { Half-Life } & \text { End Point Energy } & \text { Average Energy } \\ & 2.6 \text { years } & 545 \mathrm{keV} & 216 \mathrm{keV} \\ { }^{22} \mathrm{Na} & 288 \text { days } & 1890 \mathrm{keV} & 835 \mathrm{keV} \\ { }^{68} \mathrm{Ge} /{ }^{86} \mathrm{Ga} & 71 \text { days } & 475 \mathrm{keV} & 201 \mathrm{keV} \\ { }^{58} \mathrm{Co} & 7100\end{array}$

To obtain the two different energies needed to measure $W$ [Eq. (5)], both a ${ }^{68} \mathrm{Ge}$ source and a ${ }^{58}$ Co source would be required.

It appears feasible to use ripple injection to get energetic positrons into the plasma. Ripple injection was proposed by Jassby and Goldston ${ }^{5}$ as a way of extending neutral beam penetration. The idea is that particles trapped in toroidal field ripples at the bottom of the tokamak move approximately straight up at the curvature drift speed $\sim 5 \times 10^{4} \mathrm{~m} / \mathrm{s}$ for $200 \mathrm{keV}$ positrons in DIII-D at $2 \mathrm{~T}$. The principal was tested with a favorable result in ISX-B. ${ }^{6}$ In that experiment, central fast particle neutral 
fluxes were increased 3-10 times by the ripple coils. The ripple fields had a negligible effect on passing fast ions and thermal ions but a large depletion of perpendicular fast ions.

Schematically, a picture frame coil located just outside the TF coils would be used to produce toroidal ripple characterized by $\delta(R, Z)=\Delta \mathrm{B}_{\mathrm{T}} / \mathrm{B}_{\mathrm{T}}$. Positrons with $v_{\|} / v<\delta^{1 / 2}$ will be trapped in the ripples and will curvature drift up into the plasma. If the positron source strength is $S$ into $4 \pi$ steradians, then $\left(\pi \delta^{1 / 2} / 4 \pi\right) S$ positrons released from a source positioned between TF coil return legs will drift upward. For a $1 \mathrm{C}$ source and $\delta=1 \%, 7.5 \times 10^{5}$ positrons per millisecond will drift up into the plasma.

Since $\delta$ decreases as the positrons move up, positrons on the edge of the trapping region $v_{\|} / v=\delta^{1 / 2}(R, Z)$ will become de-trapped. Neglecting pitch angle scattering, a fraction $\delta^{1 / 2}\left(R_{0}, Z=0\right) / \delta_{\text {coll }}^{1 / 2}$ of the initially ripple trapped positrons will remain trapped at the magnetic axis. This fraction can be made small by appropriate ripple coil design.

Scattering also de-traps the particles. The slowing down and pitch angle scattering times are about equal for these energetic positrons (Fig. 1). The time to scatter across the trapping boundary will be $\sim\left[\delta^{1 / 2}(R, Z)-v_{\|} / v\right] \tau_{90^{\circ}} \simeq 50-1000 \mu \mathrm{s}$, a time somewhat longer than the $\sim 40 \mu$ s drift time to the plasma center. Hence some, but not excessive collisional de-trapping will occur.

The positrors can be put into the plasma in a $5 \mathrm{~ms}$ burst by means of a shutter on the source or preferably just pulsing the ripple coil on for only 5 ms. This latter approach has the advaritage of removing the $B_{T}$ ripple from the time in which we 


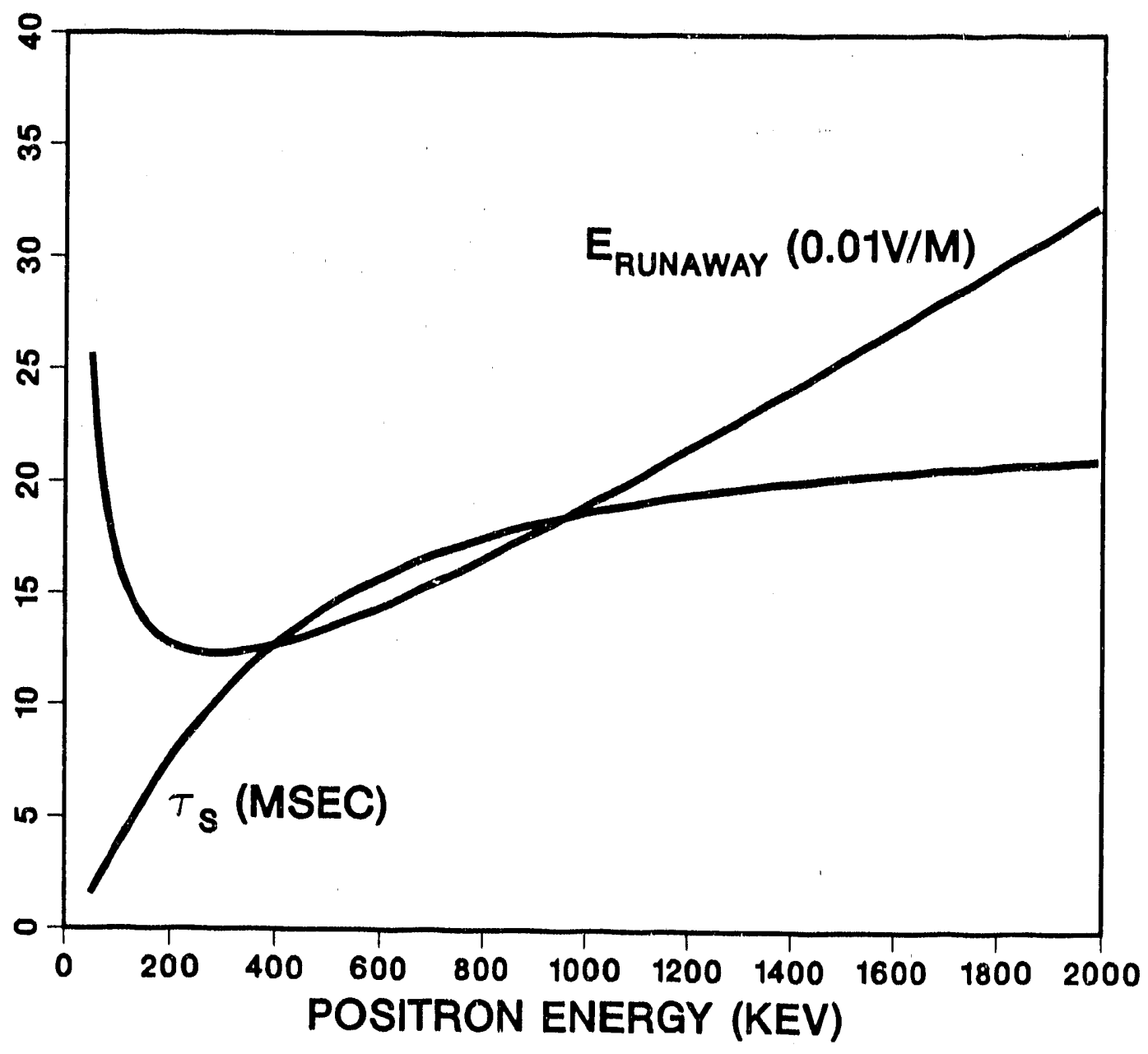

Fig. 1. Slowing down time $\tau_{s}$ and runaway electric field versus positron kinetic energy in a plasma with $n_{e}=5 \times 10^{10} \mathrm{~m}^{-3}$.

wish to see the positrons diffuse and also un-trapping all those positrons still drifting up at the time the magnet is switched off.

\section{POSITRON PLASMA INTERACTIONS}

Of course the point of injecting the positrons is to see them diffuse. Surko et al. ${ }^{3}$ modeled the diffusion of positrons assuming a diffusion coefficient $D$ of $0.5 \mathrm{~m}^{2} / \mathrm{s}$ and 
found characteristic times $\sim 30 \mathrm{~ms}$ for significant changes in the profiles. We would estimate $\chi_{E, R} \sim 1-10 \mathrm{~m}^{2} / \mathrm{s}$. However, we wish to consider things in terms of particle transport. For magnetic turbulence $D \sim \sqrt{m_{e} / m_{i}} \chi \sim 0.025-0.25 \mathrm{~m}^{2} / \mathrm{s}$ and so we expect the diffusion time scales to be $60-600 \mathrm{~ms}$, much longer than the slowing down time.

The positrons "born" into the plasma by de-trapping from the $\mathrm{B}_{\mathrm{T}}$ ripple will still be banana trapped particles. Since the banana widths are small $\left(0.8 \mathrm{~cm}\right.$ for $B_{p}=$ $\left.1 \mathrm{~T}, \mathrm{P}_{\perp}=2 \mathrm{MeV} / \mathrm{c}\right)$ it is still of interest to look at the diffusion of these banana trapped positrons. In fact the use of positrons may have some unique advantages in studying trapped particle dynamics. Based on the ISX-B result and the work in Ref. 5, we expect the ripple field to have a deleterious effect on the confinement of the banana trapped positrons. Monte-Carlo guiding center orbit calculations should be done to assess this effect, but are beyond the scope of this survey treatment of runaway positron diffusion studies.

After one $\tau_{s}$, a substantial fraction of the positrons will have scattered across the banana trapping boundary $v_{\|} / v \sim(r / R)^{1 / 2}$ onto passing orbits. They will also have lost energy. By applying a short high loop voltage, we can accelerate these positrons back to high energies. The toroidal electric field at which positron energy gain equals coulomb drag loss is given in Fig. 1. At $10 \mathrm{~V} / \mathrm{m}$, a $100 \mathrm{keV}$ positron will gain $164 \mathrm{keV} / \mathrm{ms}$. By this means we can recover a high energy population of passing particles tr observe. 
For completeness, we mention some other positron plasma interactions. Recently radial electric fields on the plasma edge of $200 \mathrm{~V} / \mathrm{cm}$ have been found in $\mathrm{I}$-mode. The $\vec{E} \times \vec{B}$ drift speed in such a field is $10^{4} \mathrm{~m} / \mathrm{s}$, large enough that positron dynamics in the edge might be of some use in studying $\mathrm{H}$-mode physics. The mean time (nonrelativistic) for direct free-free $e^{+} e^{-}$annihilation is $1 /\left(n_{e} \pi r_{0}^{2} c\right)$ (Ref. 7) where $r_{0}$ is the classical radius of the electron and is too long (2.6 seconds at $\left.n_{e^{-}}=5 \times 10^{19} \mathrm{~m}^{-3}\right)$ to be of interest except possibly in very high density devices (CIT, C-MOD, ZTH). The other annihilation process is through the formation of positronium, which is very unlikely for energetic positrons in the plasma interior. ${ }^{8}$

\section{DETECTION OF THE POSITRONS}

In a solid pellet, the annihilation time is less than $1 \mu \mathrm{s}$. The range of a $1 \mathrm{MeV}$ positron in graphite is $\sim 1 \mathrm{~mm}$, so a solid pellet injected across the flux surface will cause the immediate annihilation of the positrons which strike it. Consider a carbon pellet of diameter $\mathrm{d}=2 \mathrm{~mm}$ and a speed of $V_{p}=500 \mathrm{~m} / \mathrm{s}$. We can estimate the fraction of the positrons on a flux surface that will strike the pellet as

$$
f=\left(v_{\mathrm{pos}} \frac{d}{V_{p}}\right) /\left(\frac{4 \pi^{2} R r \kappa}{d}\right) \simeq 18 \%
$$

for typical positrons and $R \sim 1 \mathrm{~m}, r \sim 0.2 \mathrm{~m}, \kappa=1$ parameters. The annihilation $\gamma$ 's could be detected by NaI crystals viewing the pellet flight path. The time dependence of the count rate will give the positron radial distribution via the known pellet speed. 
We can estimate a count rate for a $10 \mathrm{~cm} \times 10 \mathrm{~cm}$ detector close to the pellet path with a solid angle of $\sim 100 \mathrm{~cm}^{2} /\left(4 \pi 10,000 \mathrm{~cm}^{2}\right) \simeq 10^{-3}$. From the $1 \mathrm{C}$ source we estimated $\sim 4 \times 10^{6}$ positrons ripple injected in $5 \mathrm{~ms}$. We estimate $2 / 3$ would be lost through thermalization and orbit loss. The remainder diffuse to fill the volume of the torus but the pellet recollects $\sim 18 \%$ of them. Hence $\sim 2.4 \times 10^{5}$ aniahilation events would occur and, assuming a detector efficiency of $85 \%$, we would detect $\sim 200$ of them during the $2 \mathrm{~ms}$ flight time = $f$ the pellet for an instantaneous count rate of $120 \mathrm{kHz}$. These rates are in the range to make interesting measurements. Of course, the time dependence of the annihilation $\gamma$ signal from the limiter ${ }^{3}$ will also be of interest.

\section{CONCLUSION}

The study of the diffusion of energetic positrons may enable a discrimination between magnetic and electrostatic turbulence as the cause of anomalous transport in the plasma interior. The connection between runaway positron transport is simple in the weak turbulence regime but requires supplemental information $\bar{k}_{\theta}, \mathrm{b}_{0}$, if the thermal electrons are in the strong turbulence regime. Energetic positrons might be put into the plasma by ripple injection. One of the advantages of positrons for these studies, their known initial energy spectrum, will be compromised by the slowing down and pitch angle scattering times being less than expected diffusion times. The energy spectrum of positrons in the plasma may be manipulated by means of short increases in the toroidal electric field. The radial profile of the positrons can be measured at any time after injection by the time dependence of the $e^{+}-e^{-}$annihilation $\gamma$ signal 
from an injected solid pellet. Estimated count rates appear to be in a useful range. This brief survey of the possible study of magnetic turbulence by means of energetic positrons is encouraging. Many detailed calculations remain but are possible since the physical processes described herein depend mainly on classical scattering and orbit effects.

\section{ACKNOWLEDGMENT}

It was a pleasure to acknowledge helpful discussions with C. Surko and P. Diamond. This work was supported by the U.S. Department of Energy under contract No. DE-AC03-89ER51114.

\section{REFERENCES}

${ }^{1}$ R. J. Goldston in the Symposium on the Status o 'Transport in Tokamaks, Bull. Am. Phys. So:. 34, 1909 (1989).

2 O. J. Kwon, P. H. Diamond, F. Wagner, G. Fussmann, ASDEX and NI Teams, "A Study of Runaway Electron Confinement in the ASDEX Tokamak," Nucl. Fusion 28, 1931 (1988).

3 C. M. Surko, M. Leventhal, W. S. Crane, A. Passner, F. Wysocki, T. J. Murphy, J. Strachan, W. L. Rowan, "Use of Positrons to Study Transport in Tokamak Plasmas," Rev. Sci. Instrum. 57, 1869 (1986).

4 T. J. Murphy, "Positron Deposition in Plasmas by Positronium Beam Ionization and Transport of Positrons in Tokamak Plasmas," Plasma Physics and Controlled Fusion 29, 549 (1987). 
5 D. L. Jassby and R. J. G Ildston, "Enhanced Penetration of Neutral-Beam Injected Ions by Vertically Asymmetric Toroidal-Field Ripple," Nucl. Fusion 16, $613(1976)$.

${ }^{6}$ J. Lyon, et al., in ORNU Fision Energy Division Annual Progress Report, ORNL-5843, 56 (1981).

7 P. A. M. Dirac, Proc. Comb. Phil. Soc. 26, 361 (1930).

8 E. D. Theriot, "The Hyperfine Structure of Positronium and the Decay Rate of the ' $S_{0}$ State of Positronium," Ph.D. Thesis, Yale University, (unpublished) (1967). 

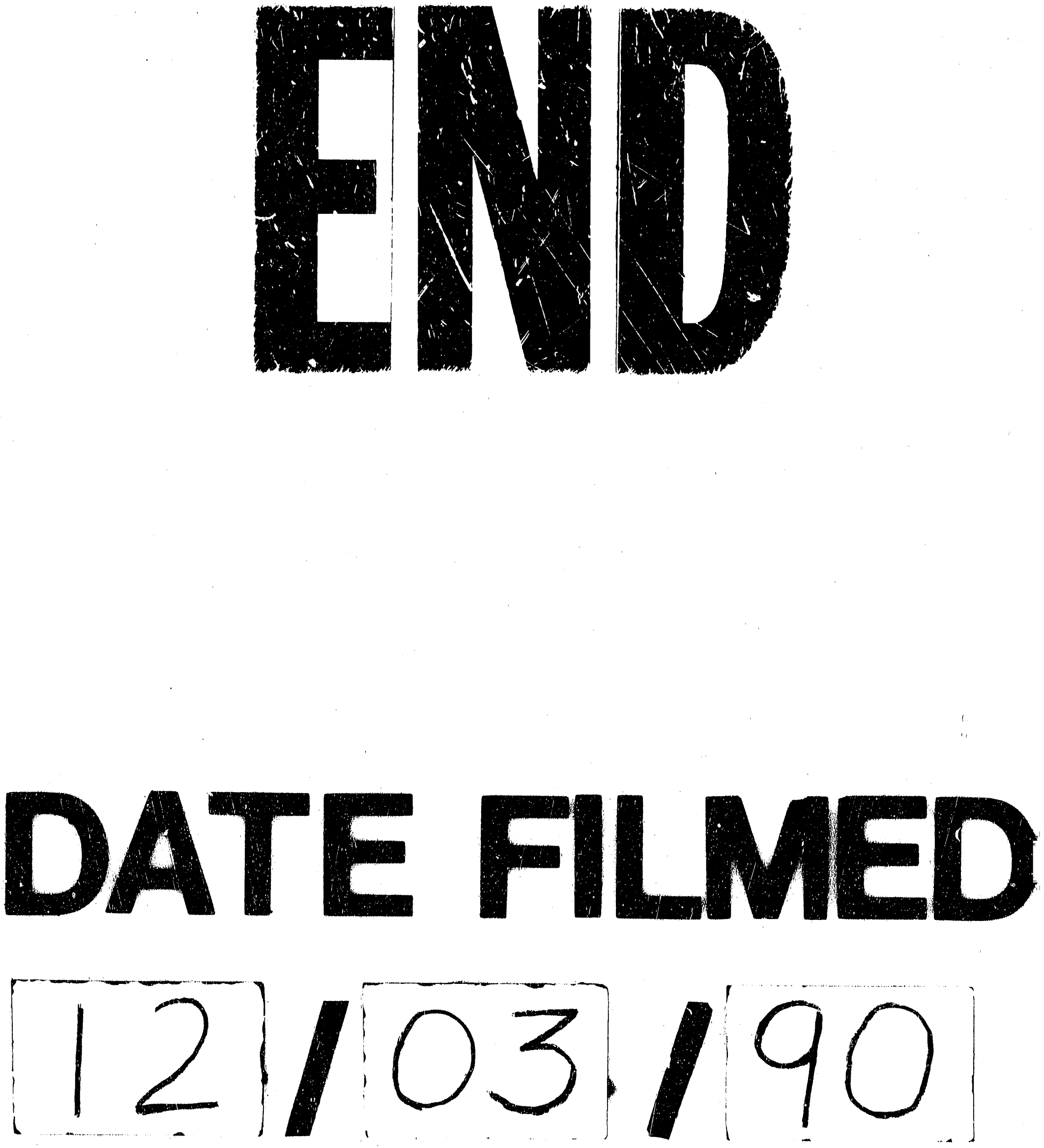
